\title{
Role of the Thalamus in Basal Forebrain Regulation of Neural Activity in the Primary Auditory Cortex
}

\author{
H. Azimi ${ }^{1}$, A.-L. Klaassen ${ }^{1,2}$, K. Thomas ${ }^{1}$, M. A. Harvey ${ }^{1}$ and G. Rainer ${ }^{1}$ \\ ${ }^{1}$ Department of Medicine, University of Fribourg, Fribourg CH-1700, Switzerland and ${ }^{2}$ Department of \\ Psychology, University of Fribourg, Fribourg CH-1700, Switzerland
}

Address correspondence to Prof. Gregor Rainer, Department of Medicine, Visual Cognition Laboratory, University of Fribourg, Chemin du Musée 5, Fribourg CH-1700, Switzerland. Email: gregor.rainer@unifr.ch

\begin{abstract}
Many studies have implicated the basal forebrain (BF) as a potent regulator of sensory encoding even at the earliest stages of or cortical processing. The source of this regulation involves the well-documented corticopetal cholinergic projections from $\mathrm{BF}$ to primary cortical areas. However, the BF also projects to subcortical structures, including the thalamic reticular nucleus (TRN), which has abundant reciprocal connections with sensory thalamus. Here we present naturalistic auditory stimuli to the anesthetized rat while making simultaneous single-unit recordings from the ventral medial geniculate nucleus (MGN) and primary auditory cortex (A1) during electrical stimulation of the BF. Like primary visual cortex, we find that BF stimulation increases the trial-to-trial reliability of A1 neurons, and we relate these results to change in the response properties of MGN neurons. We discuss several lines of evidence that implicate the BF to thalamus pathway in the manifestation of BF-induced changes to cortical sensory processing and support our conclusions with supplementary TRN recordings, as well as studies in awake animals showing a strong relationship between endogenous BF activity and A1 reliability. Our findings suggest that the BF subcortical projections that modulate MGN play an important role in auditory processing.
\end{abstract}

Key words: auditory cortex, basal forebrain, medial geniculate nucleus, neuromodulation, thalamic reticular nucleus

\section{Introduction}

The basal forebrain (BF) is a collection of nuclei including the nucleus basalis and the horizontal diagonal band of Broca (Mesulam et al. 1983; Rye et al. 1984), which exert profound neuromodulatory influences on the cortex and are involved in the regulation of diverse functions including attention, learning, and wake-sleep regulation (Baxter and Chiba 1999; Zinke et al. 2006; Herrero et al. 2008; Kang and Vaucher 2009; Quinn et al. 2010; Harris and Thiele 2011; Xu et al. 2015). The impact of BF activation on sensory neural responses has been studied extensively using reduced stimulus sets, such as drifting gratings in visual cortex (Kang et al. 2014), clicks and pure tones in the auditory cortex (Sakata 2016), or single whisker deflection in the somatosensory system (Oldford and Castro-Alamancos 2003). During natural behaviors, animals rarely encounter such steady-state reduced stimuli but must instead filter behaviorally relevant information from complex time varying sensory input. Accordingly, natural movies, i.e., dynamic visual scenes collected in natural surroundings, have proved useful for investigating sensory neural coding in the visual system. These stimuli contain time varying contrast and spatiotemporal structure and thus activate a broad range of primary visual cortex (V1) neurons. Previous studies in rat and tree shrew using naturalistic stimuli have shown that the $\mathrm{BF}$ regulates neural responses in V1 (Goard and Dan 2009; De Luna et al. 2017). During strong activation of the BF, V1 responses tend to be highly similar from trial to trial, faithfully encoding features of the sensory stimulation. Notably, enhanced reliability can be observed in V1 in both LFP and spiking activity, suggesting that it characterizes both information coding of individual neurons as well as mesoscopic activity of local circuits. During low BF activation, V1 neural activity partially uncouples from the sensory stimulation and is more strongly influenced by intracortical local brain state (Yu and Dayan 2002; Hasselmo and 
McGaughy 2004). The BF may thus trigger transitions between cortical states favoring attention to sensory inputs and states that permit integration of incoming sensory information with internal representations.

It is thought that the corticopetal projections of the BF play a major part in mediating $\mathrm{BF}$ modulation of sensory processing. These projections are segregated in the BF according to sensory modality (Laplante et al. 2005; Zaborszky et al. 2015; Kim et al. 2016), such that visual, somatosensory, and auditory areas are organized along an anterior-posterior axis with auditory projections originating in the posterior nucleus basalis (pNB), see Figure $1 B$. These projections, which consist mainly of cholinergic and some GABAergic neurons, are considered to be a major pathway by which the BF influences cortical activity (Baghdoyan et al. 1993; Edeline et al. 1994; Detari et al. 1997; Jimenez-Capdeville et al. 1997; Goard and Dan 2009; Bhattacharyya et al. 2013; Pinto et al. 2013; Anaclet et al. 2015; Xu et al. 2015). However, the BF also projects to subcortical sensory relays, providing an indirect pathway for modulating sensory neuronal activations and perception. One target of BF subcortical projections is the thalamic reticular nucleus (TRN), which forms a shell of inhibitory neurons surrounding dorsal thalamus with which it maintains strong reciprocal connections. All of the neurons in the TRN are GABAergic, and all ascending thalamocortical and descending corticothalamic fibers pass through it. The TRN is functionally organized, including topographic representations of the visual, auditory, and somatosensory modalities (Jones 1975; Shosaku and Sumitomo 1983; Conley et al. 1991; Bickford et al. 1994) and is thus ideally placed to modulate thalamocortical information transfer and prioritize among sensory modalities (Wimmer et al 2015). The BF sends both cholinergic and GABAergic projections to the TRN (Steriade et al. 1987; Hallanger and Wainer 1988; Asanuma 1989; Jourdain et al. 1989; Asanuma and Porter 1990; Bickford et al. 1994), and the action of acetylcholine (ACh) on TRN neurons has been shown to contain both excitatory (nicotinic) and inhibitory (muscarinic) components (Ben-Ari et al. 1976; McCormick and Prince 1986; Sun et al. 2013; Pita-Almenar et al. 2014; Sokhadze et al. 2019). The GABAergic projections on the other hand are expected to uniquely inhibit TRN. The subcortical projection from the BF via TRN to dorsal sensory thalamus represents a separate and possibly complementary pathway by which the BF can modulate sensory processing.

Indeed, it is well established that activity in sensory thalamic nuclei is profoundly impacted by neuromodulators including Serotonin, Norepinephrine, and ACh (Vanderwolf 1988; Steriade et al. 1990; Hirata et al. 2006). In regard to ACh, while BF does not project directly to sensory thalamus, it is reciprocally connected with the cholinergic brain stem nuclei pedunculopontine tegmentum (PPT) and laterodorsal tegmentum (LDT) that in turn send modulatory cholinergic projections to sensory dorsal thalamus and TRN (Swanson et al. 1984, 1987; Parent et al. 1988; Semba et al. 1988; Losier and Semba 1993). A number of studies have documented enhancement of reliability of neuronal signals in thalamus following the cholinergic agonist application or stimulation of aforementioned neuronal pathways, for example, in terms of reduced variance of spiking responses or enhanced entrainment to repeated stimulation (Castro-Alamancos 2002a, 2002b; Sakata 2016). These studies have generally employed reduced forms of sensory stimulation, so that whether the effects generalize to more naturalistic sensory inputs remains an open question.

Depending on their resting potential, thalamocortical projection neurons display two distinct modes of responding to sensory input, tonic and bursting, that are linked to the strength of inhibitory input from TRN (Deschenes et al. 1982; Jahnsen and Llinas 1984; Contreras et al. 1992). Unlike tonic responding, bursting activity is phasic and has a long refractory period, and its relation to sensory input becomes nonlinear. Transitions between tonic and burst modes would thus be expected to strongly affect the quality of information transfer between thalamus and cortex. While there are divergent opinions regarding the importance, or indeed even the presence, of burst mode firing in TC neurons during waking (Sherman 2001b; Llinas and Steriade 2006), there is some recent evidence that TRN plays a role in regulating attentional processes, specifically in a task that requires attending to one stimulus modality over another (Halassa et al. 2014; Wimmer et al. 2015). The extent to which these two thalamic modes occur and how they might contribute to reliability changes is a question of interest in the present study.

Taken together, the aim of our study is 2-fold: We study effects of electrical BF stimulation on sensory processing in auditory cortex and compare these findings to previous work in the visual system using similar dynamic sensory stimulation. In addition, we investigate the role of subcortical projections from the BF to the auditory thalamus via the TRN in regulating responses in the auditory pathway. We characterize and compare frequency and amplitude tuning properties to auditory inputs between auditory thalamus and cortex and discuss the contributions and interactions of corticopetal and subcortical $\mathrm{BF}$ projection systems. We complement these investigations with recordings in awake animals, examining how BF activity correlates with reliability of A1 cortical responses outside of anesthesia.

\section{Materials and Methods}

The local ethical committee on animal experimentation (canton of Fribourg) approved all experimental procedures.

\section{Animals}

A total of 10 Long Evans rats of either sex between 80 and 120 days old were used in this study. Rats were maintained on a 12/12 light/dark cycle with food and water available ad libitum.

\section{Anesthetized Recordings}

Anesthesia was induced with ketamine $(100 \mathrm{mg} / \mathrm{kg})$ and xylazine $(20 \mathrm{mg} / \mathrm{kg})$ and maintained with inhaled isoflurane $\approx 1.0 \%$ in pure oxygen. Animals were placed in a stereotactic frame with a custom head holder that fixed the position of the head without the use of ear bars (Bajo et al. 1998). A midline incision was made on the scalp, the skin and periosteum were reflected, and the temporal muscle was retracted in order to expose the skull overlying auditory cortex. Three small craniotomies were made for recordings from posterior $B F(p N B$ anterior/posterior (AP) -2.3 Medial/Lateral (ML) 3.8); TRN (AP $-3.6 \mathrm{ML}$ 3.9); and the ventral medial geniculate nucleus (MGN AP -7.8 and ML 3.4). Note that the MGN was approached at a $20^{\circ} \mathrm{AP}$ angle. A much larger craniotomy was made exposing most of the temporal lobe for A1 recordings. All recordings were made with tungsten microelectrodes (FHC, Bowdoin ME) with tip resistances of $\approx 450 \mathrm{k} \Omega$. First, the TRN electrode was lowered to a depth of $\approx 6.0 \mathrm{~mm}$, and then fine adjustments were made until auditory responses were evident. This electrode was then 
A
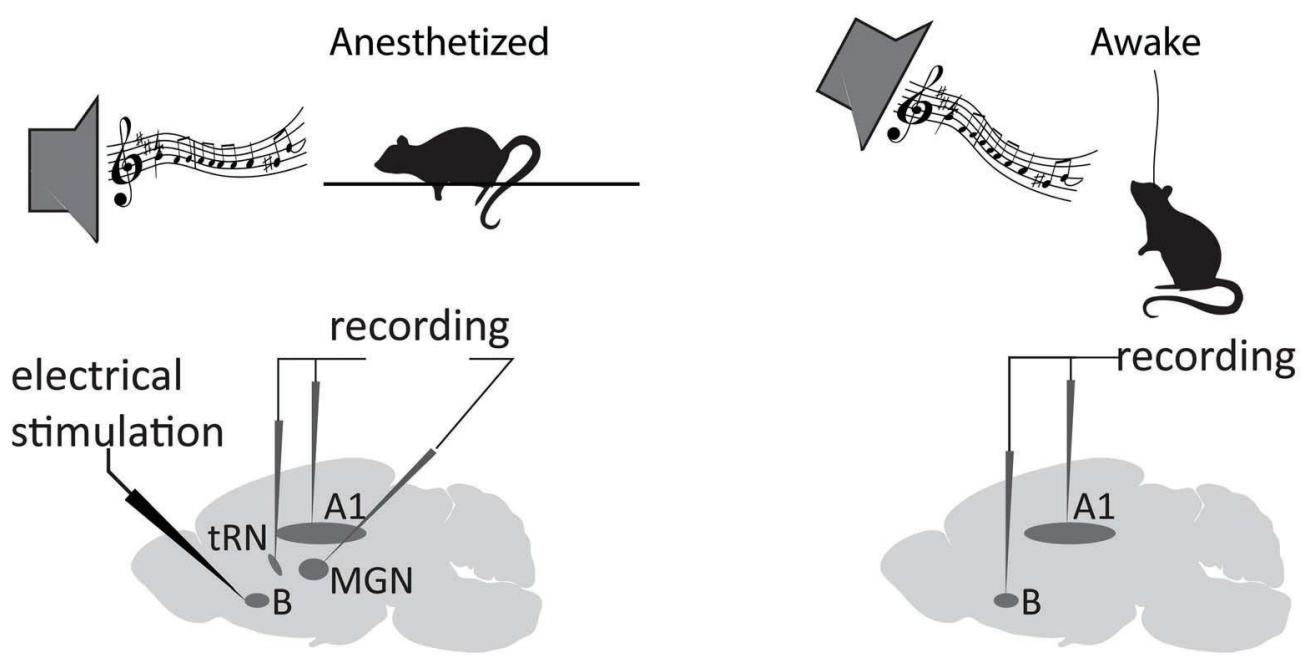

B

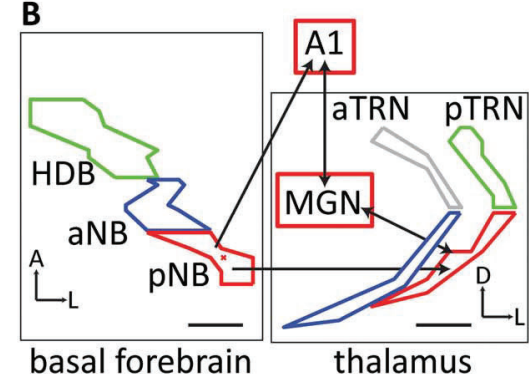

C

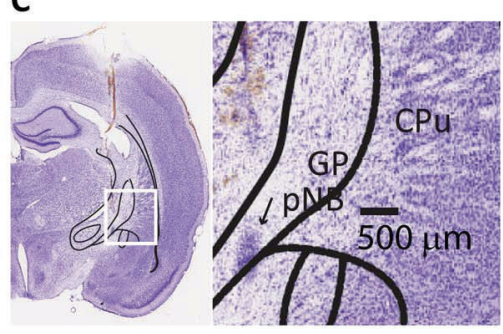

D

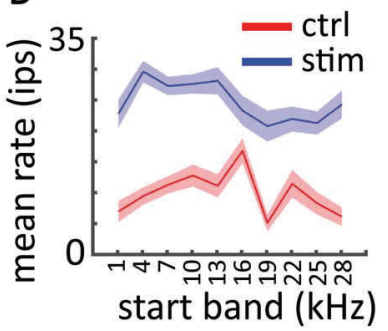

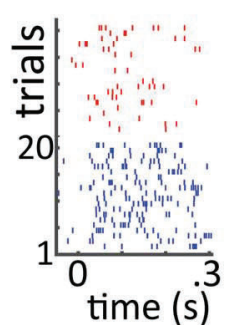

Figure 1. Experimental set up. (A) Schematic of experimental design for anesthetized (left) and awake (right) recordings. (B) Illustration of the location of modality specific BF areas (green-visual, blue-somatosensory, red—auditory), and their relationship to the auditory thalamocortical circuit, scale bar left $=1 \mathrm{~mm}$, right $=0.5 \mathrm{~mm}$. (C) Histological validation of BF stimulation site. (D) Mean response rates of an example TRN neuron to 10 different band pass noise stimuli for control (red) and BF stimulation (blue) conditions. Trial-by-trial rasters for the best frequency are shown to the right.

cemented into place using dental acrylic. Similarly, the MGN and A1 electrodes were advanced under electrophysiological guidance, using hydraulic micromanipulators (Narishige), until short latency auditory responses were observed. Finally, a monopolar stimulating electrode (FHC) was lowered to about $1 \mathrm{~mm}$ above the target location. Craniotomies were covered with 3.5\% Agarose, and the body temperature maintained with a homeostatic heating blanket (Kent Scientific) at $37^{\circ} \mathrm{C}$.

\section{Awake Recordings}

The anesthetic regimen, exposure of the skull, and electrodes were the same as for the anesthetized recordings described above. Additionally, the animals received ophthalmic ointment to prevent desiccation of the eye. Three small burr holes were made for placement of bilateral BF and left A1 electrodes (BF AP -2.3; ML 3.8/-3.8 A1 AP -5.2 ML 7). The BF electrodes were lowered to a depth of $7.5 \mathrm{~mm}$ from Bregma, and the A1 electrode was advanced until short latency auditory responses were observed, generally around $1 \mathrm{~mm}$ below the cortical surface. Electrodes were cemented into place with dental acrylic and wired to a ziff clip (TDT Apalucha) head stage, and the skull was covered with dental acrylic. A single suture was placed at both the anterior and posterior terminations of the incision. Postoperatively, the animal received a single dose of Buprenorphine $(0.05 \mathrm{mg} / \mathrm{kg})$ and Vetramil ointment was applied to the margins of the incision.
Animals received postoperative analgesia in the form of Paracetamol dissolved in their drinking water $2 \mathrm{mg} / \mathrm{ml}$ for 3-4 days following the surgery. All animals were allowed to recover for at least 7 days prior to testing.

\section{Auditory Stimulation}

Binaural auditory stimuli were delivered in an open field configuration through an electromagnetic speaker (TDT) placed $10 \mathrm{~cm}$ in front of the animal's nose. The speaker was controlled with an RZ6 auditory processor (TDT). Two main stimulus sets were used. 1) Instrumental music: we imported four instrumental music recordings (Chopin Fantasy Op.49 in F-minor; Bach Cello Suite Nr.1 Prelude; Beethoven March in D, Smetana Moldau) as MP3 files in MATLAB (2018a). We performed upsampling by a factor of three, shifting auditory stimulus structures to higher frequencies, in accordance with frequency sensitivity of the rat. Suitable music segments of $8 \mathrm{~s}$ were selected. Fourier amplitude was equalized among the four $8 \mathrm{~s}$ music segments, ensuring that all music segments possessed equal spectral content. Music stimuli were ramped (100 ms) to avoid sudden sounds at the beginning and end of the stimulus. 2) Band pass noise: Ten $300 \mathrm{~ms}$ segments of band pass noise stimuli were constructed by filtering (4-pole Butterworth ) random white noise with a constant band width of $3 \mathrm{kHz}(1-4,4-7, \ldots 28$ to $24 \mathrm{kHz})$. Stimuli were presented with a block design, such that each 
block contained a single presentation of each of the stimulus conditions. In total for both the instrumental music and band pass noise experiments, 20 blocks were presented for the control and electrical stimulation conditions. Intertrial intervals were randomized at $1 \pm 0.2 \mathrm{~s}$ and $0.2 \pm 0.05 \mathrm{~s}$ for the instrumental music and band pass noise, respectively. For the band pass noise, stimuli and additional $0.5 \mathrm{~s}$ were added between blocks where electrical stimulation was presented.

\section{Electrical Stimulation}

Monopolar constant voltage $(\approx 20 \mathrm{~V})$ electrical stimulation, using the neck muscle as a reference, was delivered to the pNB via a low impedance $(\approx 50 \mathrm{k} \Omega)$ tungsten electrode controlled by a pulsar 6i stimulator (FHC). Note that this will result in a current of $\approx 400 \mu$ A. Pulse trains had a duration of $500 \mathrm{~ms}$ and consisted of 50 and $100 \mu \mathrm{S}$ monophasic pulses, delivered at $100 \mathrm{~Hz}$, and terminated $800 \mathrm{~ms}$ prior to auditory stimulation. The stimulating electrode was lowered in $200 \mu \mathrm{m}$ steps, and electrical stimuli were delivered until both a clear desynchronization of the A1 LFP was observed, and no muscle contractions were present. Electrical stimulation was delivered prior to each of the 20 blocks of randomized trials. Thus, for the instrumental music, the interstimulation interval was $26 \mathrm{~s}$, and for the band pass noise $22 \mathrm{~s}$.

\section{Data Acquisition and Processing}

Signals for single-unit analysis were acquired through a unity gain head stage (TDT) and digitized at $24 \mathrm{kHz}$, and band pass filtered between $300 \mathrm{~Hz}$ and $8 \mathrm{kHz}$ using an RZ5 amplifier (TDT) and stored on a PC for offline analysis. Subsequent spike sorting was performed offline using Offline Sorter software (Plexon). LFP data were sampled at $2.4 \mathrm{kHz}$, and band pass filtered between 0.5 and $300 \mathrm{~Hz}$. All in all, we recorded from a total of $300 \mathrm{~A} 1,250 \mathrm{MGN}$, and 25 TRN neurons. We additionally acquired LFP recordings from 102 MGN and 102 A1 sites that were free from line noise and suitable for spectral analysis.

\section{Reliability Analysis}

All data analyses were performed using custom routines written in MATLAB (Mathworks INC). To compute trial-by-trial reliability, firing rates were calculated for consecutive $200 \mathrm{~ms}$ bins beginning at the onset of the instrumental music piece and ending $200 \mathrm{~ms}$ after the offset for a total of $8.2 \mathrm{~s}$. Each of the resulting 20 rate histograms, 1 per trial, was then cross correlated (corrcoef, MATLAB) pairwise, and we took the average of these values as the reliability score. Since neurons responded very differently to the four instrumental music pieces, we selected only the music piece, which generated the highest reliability for each neuron, i.e., the music piece that was best at driving that neuron. The same selection criteria were used for the reliability measures of the LFP, where reliability was calculated simply by pairwise cross correlations of trials using the same time segments as for the single-unit procedure.

\section{Granger Analysis}

To study directional influences in MGN and A1, we first selected only cells that showed a significant modulation of their responses to the band passed white noise stimuli (MGN $n=165$; A1 $n=145$ ). We then performed bivariate Granger causality analyses based on the spiking responses to our $8 \mathrm{~s}$ instrumental music stimuli (Schmitt et al. 2017). To create a continuous signal, we binned all the detected spikes with a bin size of $1 \mathrm{~ms}$ and applied a Gaussian filter convolution with $10 \mathrm{~ms}$ width over the binned dataset. After Z-score normalization, we fitted an autoregressive model to the time series with a maximal model order of 100 by using the MVGC multivariate Granger causality toolbox (Barnett and Seth 2014).

\section{Burst Analysis}

We counted the occurrence of bursts in MGN as those instances of interspike intervals less than or equal to $4 \mathrm{~ms}$ that were preceded by at least $50 \mathrm{~ms}$ of silence. We counted across all four instrumental music pieces, since bursting activity is stimulus independent.

\section{Naka-Rushton Analysis}

We selected only neurons that showed either significant increases or significant decreases in reliability, referred to as rel+ and rel- neurons, respectively. Firing rates in response to the broad band, 1-24 kHz pink noise stimuli, were calculated for each of 20 repeats for three stimulus amplitudes, $a=0.12,0.23$, and 0.69 , as well as during the baseline period. These data were then fit with a Naka-Rushton function as follows:

$$
r(c)=R_{\max } \frac{A^{n}}{A^{n}+A_{50}^{n}}+R_{0}
$$

where $R_{\max }$ is the peak firing rate, $R_{0}$ is the baseline firing rate, and $A_{50}^{n}$ is the semisaturation amplitude. The $A_{50}^{n}$ is inversely proportional to amplitude sensitivity with lower values reflecting increased sensitivity. We obtained good fits, Kolmogorov-Smirnov, in 70/123 MGN and 94/126 A1 neurons. We then calculated paired $t$ tests for $R_{\max }$ and $a_{50}$ for the values obtained in the ctrl and e-stim conditions. We employed NakaRushton functions because they adequately approximate neural responses for the amplitude range tested in the present study and to assure direct comparability of findings to the previous work in visual cortex that also employed these fits.

\section{Frequency Tuning}

In order to determine the effects of pNB e-stim on the frequency tuning of MGN and A1 neurons, we recorded spiking responses to 10 different auditory frequency bands, see above, with 20 repeats at each band. Firing rates were calculated for each frequency band, and a two-way ANOVA (anovan, MATLAB) was calculated for frequency band and stimulation condition (ctrl vs. e-stim). We categorized cells into those showing only a main effect of stimulation, those showing both a main effect of stimulation and a significant interaction term, and those that showed only a significant interaction term. Those cells showing a significant main effect were further subdivided into groups that showed an overall increase or decrease in firing rate after electrical stimulation.

\section{Histology}

Animals were overdosed with $200 \mathrm{mg} / \mathrm{kg}$ pentobarbital and perfused transcardially with $300 \mathrm{~mL}$ phosphate buffered saline followed by $300 \mathrm{~mL} 4 \%$ paraformaldehyde. Brains were then stored in $4 \%$ paraformaldehyde overnight and subsequently 
cryoprotected in $15 \%$ followed by $30 \%$ sucrose solution until they sank. Serial $40 \mu \mathrm{m}$ sections were cut on a sliding (Leica) microtome and stained with Cresyl Violet for microscopic examination of electrode tracts.

\section{Results}

In the present study, we characterize how activation of the $\mathrm{BF}$ impacts the reliability of neural activations in the auditory pathway to naturalistic auditory stimulation. In anesthetized studies (Fig. 1A, left panel), we electrically stimulate the BF while recording single neuron and local field potential (LFP) activity simultaneously from auditory thalamus and cortex. In awake studies (Fig. 1A, right panel), we examine correlations between gamma activity in the $\mathrm{BF}$ and reliability of LFP responses in the auditory cortex.

We begin by describing results obtained under isoflurane anesthesia, where we electrically stimulated the auditory BF (pNB), activating neural elements around the tip of the electrode largely irrespective of cell type. We used Nissl-stained coronal brain slices to verify the position of stimulation and recording sites. An example is provided in Figure $1 C$, showing the electrode tip position during electrical pNB activation that we marked using coagulation upon termination of the experiment. The electrode tip was within $200 \mu \mathrm{m}$ of the pNB nucleus, providing anatomical validation of accurate positioning in relation to the target brain structure. As noted above, the TRN plays an important role in the neural circuit connecting BF to MGN, acting as the main relay between these structures. We performed validation recordings and obtained activity of 25 TRN neurons during electrical pNB activation in order to confirm an involvement of the TRN in mediating BF activation effects on the auditory thalamus. For these recordings, we used short segments $(0.3 \mathrm{~s})$ of band pass noise (see Methods) to examine frequency tuning of the recorded neurons. Of the 25 TRN neurons, $80 \%$ (20/25) was tuned for sound frequency and $36 \%(9 / 25)$ modulated their activity following the pNB electrical stimulation (two-way ANOVA main effects of frequency or e-stim, $P<0.05)$. Of the TRN neurons that showed a main effect of our stimulation, four decreased their firing rate and five increased their firing rate. The activity of an example TRN neuron is shown in Figure 1D, exhibiting selectivity for both sound frequency and electrical stimulation. These data indicate that we accurately target the auditory segment of the TRN, and that TRN activity is indeed modulated by pNB electrical activation consistent with anatomical projections. Following transient pNB activation (100 Hz monophasic pulses for $0.5 \mathrm{~s}$, terminating $800 \mathrm{~ms}$ prior to the auditory stimulus), we simultaneously recorded single-unit activity and LFP in auditory cortex (A1) and auditory thalamus (MGN) during auditory stimulation with sounds containing complex and dynamic spectral signatures. Comparing neural responses in these structures to control conditions without electrical stimulation allows us to examine how BF activation impacts information transmission and encoding in the auditory pathway.

We next assessed how BF stimulation affected reliability of LFP responses in A1 and MGN. Prior to initiating recordings we advanced our pNB electrode from $1 \mathrm{~mm}$ above target in $200 \mu \mathrm{M}$ steps until electrical stimulation both desynchronized the cortical LFP, and no muscle contractions were present. Indeed, electrical stimulation resulted in a significant decrease in the integral of the FFT between 1 and $10 \mathrm{~Hz}$ for all of our animals $(P<0.01$ paired $t$ test), and Figure 2A shows an example. We obtained LFP recordings of sufficient quality for quantitative analysis from 102 sites in A1 and 102 sites in the MGN during presentation of instrumental music segments. In Figure $2 B$, we show the spectrogram of one of the employed music segments (Chopin Mazurka), illustrating multiple spectrally broad transients and a spectrum generally dominated by lower frequencies. In both conditions, high amplitude LFP deflections, so-called depth-negative waves, occurred frequently during auditory stimulus presentation. In cortex, depth-negative waves are associated with neural spiking discharge and represent periods of brain activation. In the control condition, depth-negative waves tended to occur somewhat haphazardly at unpredictable times during the trial. Following pNB stimulation, LFP depthnegative waves occurred more reliably at particular times during auditory stimulus presentation from trial to trial. We quantified reliability by computing the average correlation coefficient between all trials of LFPs collected under the same conditions. We then used the preferred music segment for each LFP site for further analyses (see Methods). For the present example, reliability increased from 0.053 in the control condition to 0.27 in the pNB stimulation condition, consistent with enhanced entrainment of the A1 LFP to the spectrotemporal characteristics of the auditory stimulus. Indeed, pNB stimulation enhanced LFP reliability across the population of recorded A1 sites (paired $t$ test: $P<0.01$ ), with significantly more sites showing reliability increases versus decreases ( 65 vs. 37 sites, $\chi^{2}$ test: $P<0.05$ ), see Figure $2 \mathrm{C}$. Note that $\mathrm{A} 1$ reliability enhancements only occurred during auditory stimulus presentation and were not seen in a control period prior to auditory stimulation in the absence of auditory input (paired $t$ test: $P>0.1$ ). For the pNB stimulation condition, we confirmed that LFP responses were not only similar to each other from trial to trial but also closely related to the auditory stimulus. Specifically, we found that the mean LFP response was significantly positively correlated to the auditory stimulus envelope as estimated from the Hilbert transform across recording sites, $n=102$ (range of correlations: $0.05-$ 0.65 ; mean correlation $0.41 ; P<0.01$ ). The optimal temporal lag between LFP and auditory envelope was $-15 \mathrm{~ms}$, corresponding well to the latency of the A1 auditory response. No effects of pNB stimulation on reliability were evident in the MGN (see Fig. 2D), which unlike cortex does not possess dendrites with consistent directional organization. The MGN LFPs thus did not exhibit prominent stimulus-entrained deflections in either the control or pNB stimulation conditions. LFP reliability values, i.e., average trial-by-trial correlations, did not exceed a value of 0.1 in either condition, suggesting that MGN LFPs exhibited minimal entrainment to our auditory stimuli, see Figure 2E. Our findings extend previous observations (De Luna et al. 2017), in visual cortex to auditory cortex A1, suggesting the generality across sensory modalities of $\mathrm{BF}$ activation related to enhancement of cortical LFP reliability.

In visual cortex, BF stimulation enhances trial-to-trial reliability not only of LFPs but also of single-neuron activity (Goard and Dan 2009; De Luna et al. 2017). We therefore proceeded to examine single-neuron reliability for our population of 250 MGN and 300 A1 neurons. Spike trains for an example A1 unit in response to one $8 \mathrm{~s}$ instrumental music segment are shown in Figure 3A. Visual inspection suggests spiking responses occur more reliably after pNB stimulation, see, for example, the transient response at $6 \mathrm{~s}$. To quantify this effect, we computed reliability as the average cross correlation between spike trains binned at $200 \mathrm{~ms}$ using the preferred music segment for each neuron (see Methods). For the example A1 unit in Figure 3A, we observed a reliability increase in $0.06-0.38$ following the 

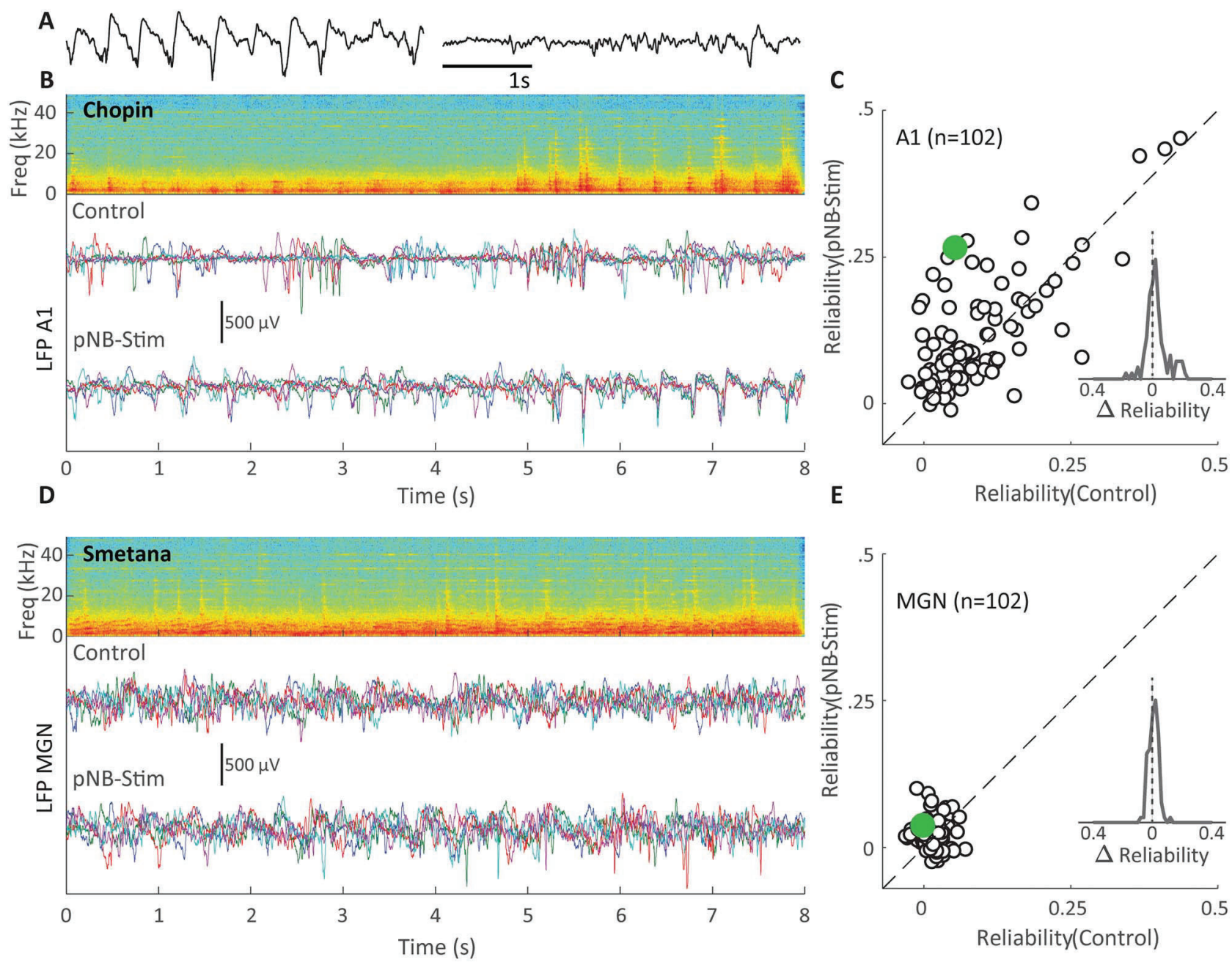

Figure 2. (A) LFP recordings from A1 before (left) and after electrical pNB stimulation (right) showing stimulus induced cortical desynchronization, these records include no auditory stimulation. (B) The spectral content over time of one of the $8 \mathrm{~s}$ classical music pieces (Chopin Mazurka), top. Note the multiple spectrally broad transients during the last $4 \mathrm{~s}$ of the music. Middle and bottom traces show five trials each of the A1 LFP in response to the music piece depicted in the spectrogram before and after pNB stimulation, respectively, auditory stimulus onset at time 0 . (C) Reliability in the control condition is plotted against reliability in the pNB stim condition for all recording sites, green symbol is the exemplar data point corresponding to the LFP traces in B, inset shows the distribution of reliability change (reliability stim reliability $\operatorname{ctrl}=\Delta$ reliability) across the population. (D) is as for A, but for recordings from the MGN in response to Smetana's Moldau. (E) As C but for MGN

pNB stimulation, consistent with robustly enhanced entrainment of this unit to the auditory stimulus. This enhancement was also evident across the population of A1 neurons (paired $t$ test considering all recorded A1 units: $P<0.01$ ), see Figure $3 B$. As is evident in the scatter plot, we found A1 units showing both increases and decreases in reliability following the pNB stimulation. We assessed for each unit whether its reliability change was significant (see Methods) and found 79 A1 units showing increases and 44 units showing decreases in reliability $\left(\chi^{2}\right.$ test: $\left.P<0.05\right)$. We repeated this analysis for the MGN; an example unit is shown in Figure 3C. It is evident that responses in both control and pNB stimulation conditions contain robust stimulus-triggered transient activations, with this unit exhibiting strong trial-to-trial reliability in both conditions ( $0.32 \mathrm{vs.} 0.37$, respectively). Consistent with this example unit, we observed no significant change in reliability in the MGN population (paired $t$ test: $P>0.1$ ), see Figure 3D, with a similar number of neurons showing increases and decreases in reliability following the pNB stimulation (63 vs. $63: \chi^{2}$ test: $P>0.1$ ). Interestingly, the overall reliability was substantially higher in MGN than in A1. For example, while only $4 \mathrm{~A} 1$ units exceeded a reliability of 0.4 in the control condition, 48 MGN units surpassed this arbitrary threshold. Indeed, across all recorded units in the control condition, MGN units were on average more reliable than A1 units $\left(r_{\mathrm{A} 1}=0.07, r_{\mathrm{MGN}}=0.18\right.$; unpaired $t$ test: $\left.P<0.01\right)$. In a subset of recordings, $n=15$, we waited at least $10 \mathrm{~min}$ following the pNB stimulation presented the instrumental music and measured reliability as a "washout" procedure. The results, as seen in Figure 3E, show that following this waiting period reliability was again reduced to near baseline levels. In order to test whether changes in reliability were related to firing rate modulations, we performed two control analyses. First, we calculated reliability using a range of different bin sizes $(5,10,15, \ldots 300 \mathrm{~ms})$. We found that pNB stimulation significantly increased reliability over control values for every bin size. Second, we equalized spike train pairs by randomly deleting spikes from the spike train with the higher firing rate (Goard and Dan 2009). Reliability results remained similar after this procedure, indicating that increases 
in reliability were not due to changes in firing rate. Thus, pNB stimulation enhanced overall reliability in A1 but not in the MGN, and these results were not due to generalized rate changes but rather to an enhanced representation of the auditory stimuli in A1.

In order to understand how pNB stimulation affected the directional coupling between MGN and A1 during auditory stimulation, we calculated Granger causality of the spike trains in both areas during the $8 \mathrm{~s}$ instrumental music pieces before and after pNB stimulation, Figure $3 F$. We first selected only those A1, $n=145$, and MGN, $n=165$, units whose responses to the band pass noise stimuli were significantly modulated by pNB stimulation. A two-factor ANOVA for the effects of pNB stimulation and directional (MGN $\rightarrow$ A1 vs. A1 $\rightarrow$ MGN) interactions showed only a significant effect of pNB stimulation, $P<0.001$. Post hoc analysis revealed this to be due to an increased Granger causality between MGN and A1, consistent with the idea that pNB stimulation increases the thalamic drive to A1.

Each brain area contained populations of neurons with increasing as well as decreasing reliability. We therefore proceeded to investigate whether these two populations possess different characteristics in terms of their tuning to sound amplitude and frequency content. To examine how spiking activity in MGN and A1 depended on sound amplitude, we used broadband (1-24 kHz) auditory noise at three amplitudes $(0.12,0.23$ and 0.69$)$. Note that the amplitude dependence was studied in independent data sets from those of the reliability measurements. We fit Naka-Rushton functions, see Methods, to the rate-amplitude data, extracting responsivity $\left(R_{\max }\right)$ and halfmaximum amplitude $\left(a_{50}\right)$ parameters for each neuron. Note that the $a_{50}$ parameter reflects sound sensitivity, with lower values indicating higher sensitivity. We performed separate fits for control and pNB stimulation data and compared populations with significantly increasing versus decreasing reliabilities (rel+, rel-). Results for a single A1 rel+ unit are shown in Figure $4 \mathrm{~A}$, illustrating enhancement of firing rate $\left(R_{\max } 33 \mathrm{vs}\right.$. $68 \mathrm{sp} / \mathrm{s})$, and increased sound sensitivity ( $a_{50} 0.12$ vs. 0.04$)$. Examining the data for the population of $48 \mathrm{rel}+$ and 32 rel- A1 neurons with adequate goodness of fit (total $n=190$, see Fig. $4 B, C$ ), we observed significantly increased $R_{\max }$ and decreased $a_{50}$ values (paired $t$ tests: $P<0.01$ ), consistent with the single-unit example. Thus, rel+ A1 units responded both more strongly and with greater sensitivity to auditory input following the pNB activation. This was not the case for the population of 32 A1 units, whose $R_{\max }$ and $a_{50}$ parameters were unaffected by pNB activation (paired $t$ tests: $P>0.1$, see Fig. $4 D$ ). Note that rel- neurons were not simply unresponsive to the auditory stimulus ( $R_{\max }{ }^{\text {rel-}}$ : $33.2 \pm 8.4$ vs. $R_{\max }{ }^{\text {rel+ }}: 25.2 \pm 4.2$, unpaired $t$ test: $P>0.1$ ). Similar to the rel+ A1 population, the rel+ MGN population also exhibited enhanced responsivity and sensitivity following the pNB activation. Data for an example MGN unit are shown in Figure 4E, illustrating an increase in $R_{\max }$ and a decrease in $a_{50}$, as was indeed also observed in the rel+ MGN population (paired $t$ tests: $P<0.01$, see Fig. $4 F, G$ ). Compared with the rel+ MGN population, the rel- MGN units also exhibited a significant increase in $R_{\max }$ (paired $t$ test: $P<0.01$ ). Interestingly, the rel- MGN neuron's $a_{50}$ parameter exhibited a significant increase following the pNB activation, thus showing a decreased sensitivity to auditory input. Additionally, we estimated $R_{\max }$ for the entire population of thalamic and cortical units regardless of reliability modulation. We found that for MGN, $R_{\max }$ was slightly but significantly increased ( $\Delta$ rate $=0.68 \mathrm{sp} / \mathrm{s}, n=194$ [good fits only], paired t test: $P<0.01$ ) consistent with findings in the rel+ and rel- population. For the general population In $\mathrm{A} 1$, we observed no significant change in $R_{\max }$ ( $\Delta$ rate $=$ $0.03 \mathrm{sp} / \mathrm{s}, n=190$, paired $t$ test: $P>0.1$ ), see Figure $4 \mathrm{H}$. In summary, neurons in both thalamus and cortex that specifically increased their reliability also increased both overall responsiveness $\left(R_{\max }\right)$ and sensitivity to auditory stimuli $\left(a_{50}\right)$. Increased sensitivity implies that neurons will be more likely to respond to particular spectral stimulus features of the instrumental music on any given trial, contributing to the overall enhancement in reliability following the pNB activation. However, neurons with decreased reliability exhibited different characteristics in thalamus and cortex, indicating differential functional modulation of these two brain structures. We wanted to know if the changes we observed in the reliability of neural signals in the auditory pathway might be related to thalamic firing mode. Sensory thalamus is known to possess two distinct firing modes, known as tonic and burst mode (Sherman 2001a; Llinas and Steriade 2006). According to established criteria, thalamic burst mode is characterized by a succession of at least two spikes with an ISI under $4 \mathrm{~ms}$, preceded by a quiet period of $50 \mathrm{~ms}$ (Llinas and Steriade 2006). Using this estimate, methods, we found a low occurrence of thalamic bursts suggesting that MGN was generally not in burst mode during our recordings under light isoflurane anesthesia. On average, we observed $0.14 \pm 0.21$ bursts per second (mean, SD), with maximum values in the population reaching at most one burst per second. MGN units thus spent little time in burst mode during our recordings, and this was unchanged by pNB stimulation ( $t$ tests, $P>0.1$ ). This indicates that the effects described in this report are unrelated to changes in thalamic firing mode, since under our experimental conditions the thalamus was mostly in tonic mode.

We next wanted to know how pNB stimulation affected the frequency tuning of MGN and A1 neurons. We reasoned that investigation of frequency tuning might help delineate the underlying mechanism that leads to the changes in response reliability. We therefore assess whether $\mathrm{BF}$ stimulation leads to a general gain modulation of firing rate independent of auditory selectivity, or whether these effects might be restricted to specific frequencies. To test this, we presented band passed noise stimuli spanning most of the rat's audible frequency range (1-24 kHz). Specifically, we presented 10 band passes with a constant width of $3 \mathrm{kHz}(1-4 ; 4-7, \ldots 28-31 \mathrm{kHz})$; additionally, we included a broadband, $1-24 \mathrm{kHz}$, stimulus condition. First, a twoway ANOVA with factors for band pass and electrical stimulation showed that all cortical $(n=300)$ and thalamic $(n=250)$ neurons showed significant frequency tuning. In A1 38\% or $115 / 300$ neurons showed only an effect of pNB stimulation, meaning that these neurons significantly changed their firing rates over a broad range of frequencies, Figure $5 \mathrm{~A}$ summarizes these results and an example neuron for A1 is shown in Figure 5B. A smaller population of A1 neurons showed either both an effect of pNB stimulation and significant interaction terms, or only significant interactions indicating that for these neurons pNB stimulation modulated responsiveness in a frequency-dependent manner, Figure 5A,C. Similar results were found in MGN with $42 \%$ or $106 / 250$ neurons showing only a main effect of stimulation, Figure 5D,E, and smaller groups showing significant, interaction terms, Figure 5D, F. Interestingly we found a higher proportion of neurons in MGN $(165 / 250,66 \%)$ were modulated by pNB stimulation than in $\mathrm{A} 1(145 / 300,48 \%), \chi^{2}$ test; $P<0.01$, indicating that pNB stimulation had a stronger modulatory effect in this area. For neurons exhibiting interactions in MGN and A1, we observed effects across all auditory frequency bands tested, with 
A

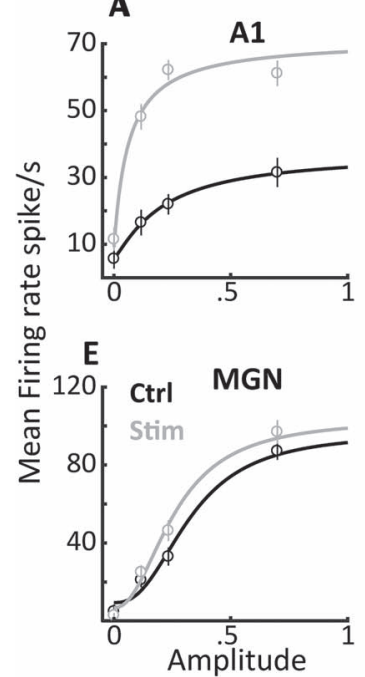

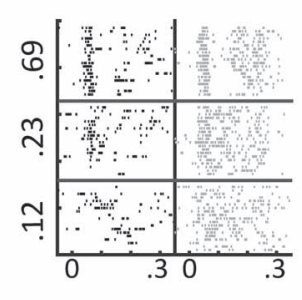

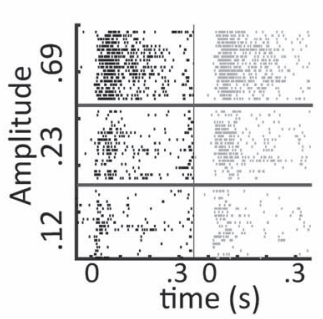

B

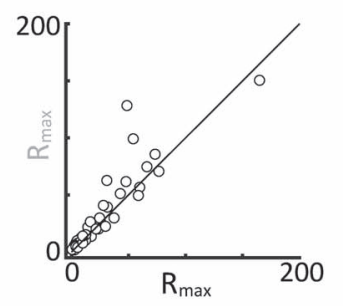

F

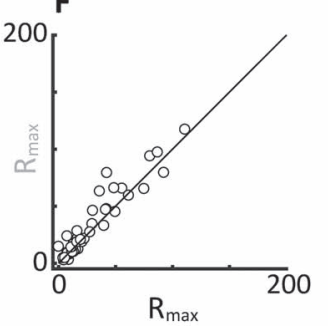

C

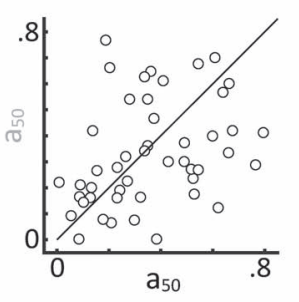

G

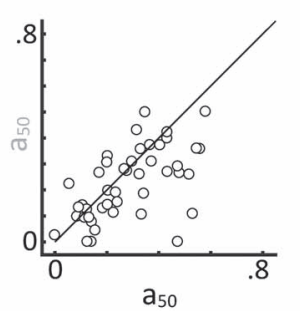

D

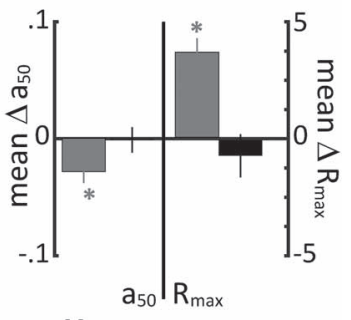

H

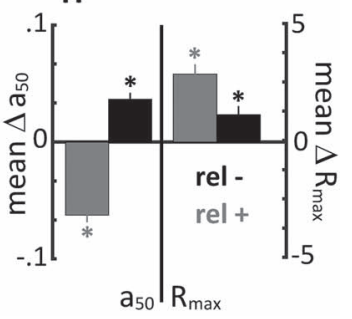

Figure 4. Example of fitted Naka-Rushton functions for single A1 and MGN neurons is shown in (A) and (E), respectively, with trial-by-trial rasters over the three stimulus amplitudes shown at right. (B, F and C, G) Show the $R_{\max }$ and $a_{50}$ values for the A1 and MGN rel+ populations in control (red) versus pNB stimulation (blue) conditions. ( $D$ and $H$ ) Compare the difference scores (pNB stim—control conditions) for the $a_{50}$ values left, and $R_{\max }$ values, right in the two populations (rel-, black; rel+, gray) for the populations of A1 and MGN neurons.

the preferred frequency changing $22 / 30$ and $33 / 59$ of neurons in A1 and MGN, respectively.

Thus, electrical stimulation of pNB had a significant impact on the responsiveness to our band pass noise stimuli, and these effects could be frequency specific. This result may be due to the position of our recording electrodes, i.e., those cells showing a significant interaction term may have receptive fields aligned with the tonotopy of pNB stimulation. We further subdivided cells showing an overall increase versus an overall decrease in firing rate and found that pNB stimulation was equally likely to upregulate as downregulate responses under these conditions. This diversity of effects on firing rate may be due to the presence of both GABAergic and Cholinergic projections from the pNB to both TRN and to A1. We wanted to know whether a relation between the endogenous activation of the BF and reliability of auditory signals also existed in the awake state. We therefore recorded activity in $\mathrm{pNB}$, while animals were passively listening to the same instrumental music segments used in the anesthetized experiments (see Methods for awake recordings). We have previously documented prominent gamma oscillations in the $\mathrm{BF}$ whose amplitude varies with the behavioral state of the animal (Nair et al. 2016; Nair et al. 2018). Our current recordings from the pNB also revealed strong gamma oscillations, as shown for an example animal in Figure $6 \mathrm{~A}$. The amplitude of the gamma oscillations serves as an indicator of BF activity, which we can relate to reliability of auditory cortex (A1) responses. We proceeded to compute a time course of A1 reliability, by considering local similarity of five A1 responses to the same auditory stimulus recorded at nearby times. This allowed us to track how reliability evolved over the course of the experiment (see Methods). As shown for an example dataset (Fig. 6B), A1 reliability was not stable but instead fluctuated considerably during the course of the session. Plotting pNB gamma power for the same segments, we observed that periods of high pNB gamma tended to be accompanied by low cortical reliability. This was confirmed by a significant negative correlation between these signals $(r=-0.71, P<0.01$, see Fig. $6 \mathrm{~B})$. Across the recordings, we found that the correlation of the regression was significantly negative $(P<0.01)$, Figure $6 C, D$, supporting the hypothesis that endogenous activation levels of the BF pNB nucleus had an impact on reliability of cortical A1 auditory responses.

\section{Discussion}

Our findings indicate that activation of the BF causes an enhancement of the reliability of neural responses in the primary auditory cortex (A1), in terms of increasing the trialby-trial correlation of spiking as well as LFP responses. Similar effects have previously been observed in the primary visual cortex (Goard and Dan 2009; De Luna et al. 2017), suggesting that the upregulation of reliability is a general property of the BF across sensory modalities. Interestingly, enhancements in cortical response reliability have also been documented in the somatosensory cortex (Hirata and Castro-Alamancos 2011), albeit following the stimulation of the cholinergic brain stem nuclei PPT/LDT and not the BF as is the case in the studies mentioned above. In that study, cholinergic brain stem stimulation as well as cholinergic agonist infusion into sensory thalamus was shown to enhance the reliability of membrane potential fluctuations to whisker stimulation in rodent barrel cortex. Both PPT/LDT and the BF contain a considerable number of cholinergic projection neurons, and while PPT/LDT does not target cortex, they do maintain a significant cholinergic projection to the thalamus encompassing both the TRN and sensory thalamic structures (Levey et al. 1987), as well as sharing reciprocal connections with BF (Swanson et al. 1984, 1987; Parent et al. 1988; Semba et al. 1988; Losier and Semba 1993). This suggests that the reliability enhancements across the three sensory modalities may involve convergent pathways onto the TRN from PPT/LDT and BF, as well as a contribution of direct PPT/LDT projections to the sensory thalamus. The enhanced A1 reliability we observed is consistent with the previous observations of reduced Fano factor and enhanced predictability of spiking responses based on the auditory stimulus envelope in A1 following the tail pinch in the rat (Marguet and Harris 
A

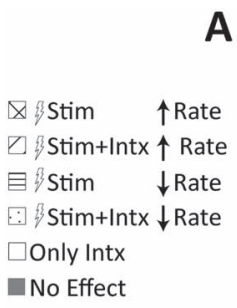

D
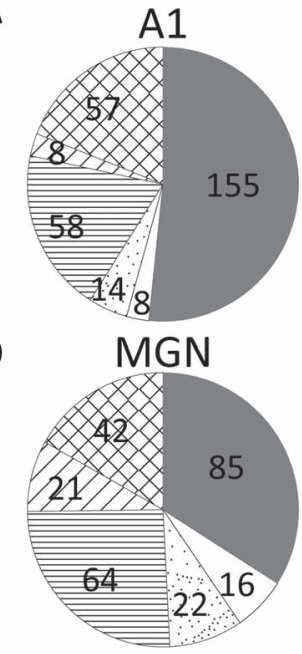
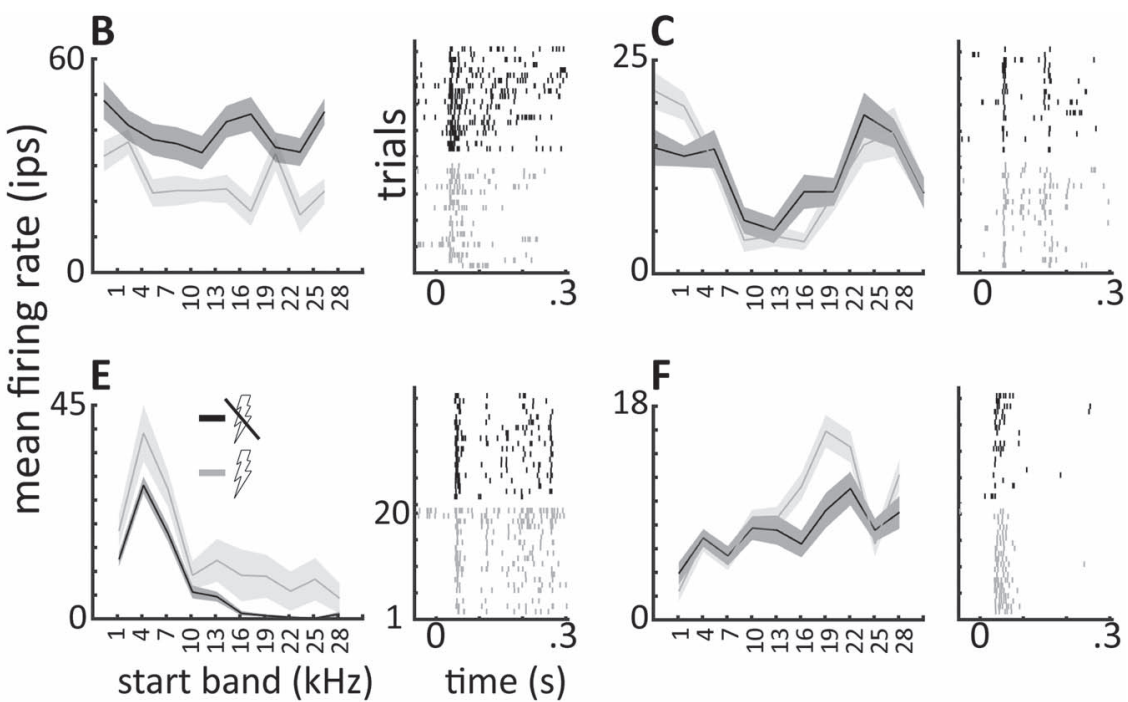

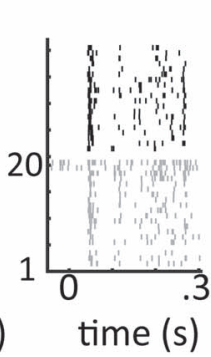

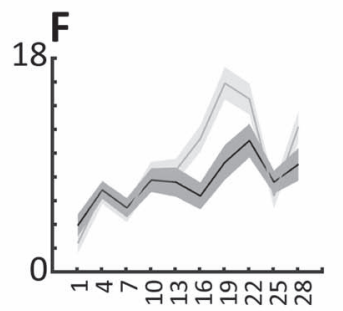

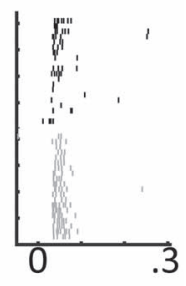

Figure 5. A two-factor ANOVA (Stim = pNB stimulation, Intx = interaction) was used to compute the effect of pNB stimulation on firing rate across 10 frequency bands in A1 (Top Row) and MGN (Bottom Row) neurons. (A and D) Show the distribution of significant effects across these populations in A1 and MGN. The most common effect of pNB stimulation was a band-pass independent decrease $(B, A 1)$ or increase $(E, M G N)$ in firing rate. The rasters to the right show the trial-by-trial responses $(n=20)$ for the preferred frequency of that neuron in control (red) and pNB stim (blue) conditions. Additionally, neurons could also increase or decrease their firing rate only for specific band pass stimuli as illustrated in (C and F) for A1 and MGN neurons, respectively.

A

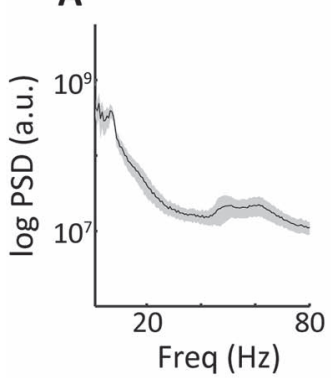

B

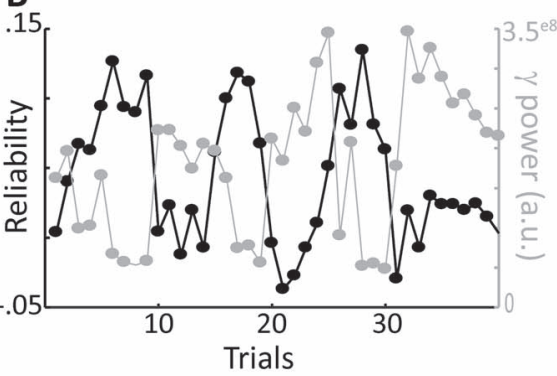

C

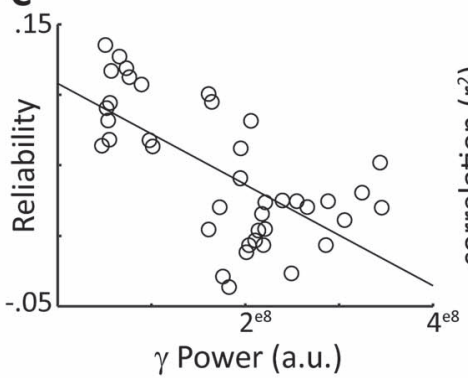

D

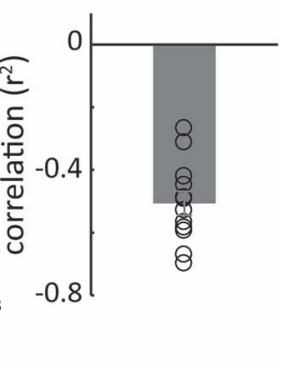

Figure 6. The relation between nucleus basalis (pNB) gamma activity and A1 reliability in the awake state. (A) Average of the fast-Fourier transforms of recorded LFPs, showing gamma band $(40-80 \mathrm{~Hz}$ ) oscillation increase in the pNB. (B) The A1 reliability (blue line) versus the pNB gamma power (green line) over time in an example animal listening to a segment of Moldau. (C) Summary of the pNB gamma power versus the A1 trial-by-trial reliability and their least-square regression (gray line) over the population of recording sites. (D) Correlation coefficients $(n=12)$ of the linear regression lines between the A1 reliability and the NB gamma band power across all individuals and conditions (three animals, four instrumental music pieces).

2011). This intervention is thought to produce similar effects as BF electrical stimulation although these effects are not identical and tail pinch recruits a more extended network involving additional brain nuclei and neurotransmitters (Smith et al. 1997; Boucetta and Jones 2009). Along similar lines, a reduced Fano factor and enhanced temporal modulation of spiking activity to click train auditory stimuli have also been documented following the BF electrical stimulation (Sakata 2016), although the stimulation sites were more anterior than in our study. Indeed, Sakata and colleagues also demonstrated that spike count correlations between pairs of A1 neurons during spontaneous activity were reduced following the $\mathrm{BF}$ stimulation, increasing the ability of individual neurons to signal-independent acoustic information and contributing to enhanced reliability. It is intriguing that this previous study used urethane anesthesia, potentiating nicotinic ACh Receptor ionic currents, while the present study employed isoflurane, an inhibitor of these same ion channels. This might suggest that effects of BF stimulation on reliability involving cholinergic neuromodulation may depend specifically on muscarinic ACh receptors. Indeed, muscarinic receptors are critical regulators of increased activity at both the thalamic and cortical levels. In rat, TRN and MGN neurons express both nicotinic and muscarinic receptors (Rotter et al. 1979; Clarke et al. 1985). In mouse TRN, optogenetic stimulation of cholinergic BF axons gives rise to fast, short latency nicotinic EPSCs and slow, sustained, muscarinic IPSCs (Sun et al. 2013; Pita-Almenar et al. 2014), whereas in the MGN of the guinea pig, iontophoretic application of ACh leads to an initial hyperpolarization followed by a long-lasting muscarinic-dependent depolarization. Thus, longterm effects of ACh attributable to muscarinic activation can directly depolarize thalamocortical projection neurons as well as attenuate TRN-mediated inhibition, leading to increased excitability and perhaps a transition to tonic firing (McCormick and Prince 1987; McCormick 1993). However, nicotinic receptors are prominently expressed on terminals of thalamocortical and corticothalamic afferent fibers (Lavine et al. 1997; Kawai et al. 2007; Sottile et al. 2017). By upregulating excitatory reciprocal 
drive between thalamus and cortex, nicotinic receptors are strong candidates for upregulating spiking activity, as well as possibly reliability in the auditory or other sensory systems. In the cerebral cortex, the diversity of cell type-specific effects of ACh is more complex (see Munoz and Rudy 2014 for a review). Glutamatergic neurons in the cerebral cortex show cell typedependent responses to ACh. Layer IV spiny stellate cells show a muscarine-dependent, sustained, hyperpolarization, whereas pyramidal neurons across cortical layers, and areas show a long-lasting, muscarine-dependent activation (Eggermann and Feldmeyer 2009). GABAergic neuronal responses to ACh also appear to be cell type specific. Importantly, the primary effect of ACh on PV basket cells is a muscarine-dependent synaptic depression. As these cells provide a major source of inhibition to both the spiny stellate cells of layer IV and to pyramidal neurons in layers II/III and V/VI, the depression of these synapses would result in a decrease in feed forward inhibition from the thalamus, as well as a disinhibition in superficial cortical layers (Kruglikov and Rudy 2008). Nicotinic receptors are also present on all GABAergic neurons in layer I of cortex (Christophe et al. 2002; Gulledge et al. 2007), and their activation results in the transient depolarization of these cells, which has been shown to inhibit nonpyramidal layer II/III neurons, potentially serving to disinhibit downstream glutamatergic cells. Similarly, bipolar VIP neurons in layer 2/3 show excitatory response to both muscarine and nicotine, as these cells preferentially target layer $2 / 3$ somatostatin neurons, which may further disinhibit the network. However, GABAergic somatostatin neurons across cortical layers display a sustained muscarinic-dependent activation. Thus, while the general picture of ACh action in the cortex is one of the increased excitabilities, the interactions between different neuronal types and the compartmentalization of different ACh receptor subtypes could provide the scaffolding for disparate cholinergic effects, perhaps tuned to behavioral demands.

While a majority of A1 neurons significantly increased their reliability, we also observed a substantial number of neurons with decreased reliability. Interestingly, these two populations differed in terms of their sound amplitude tuning, as we quantified by estimating sensitivity and responsivity parameters. Sensitivity was enhanced for the reliability-increasing neurons, whereas it was unchanged for the reliability-decreasing neurons. Increased sensitivity indicates that a neuron is more likely to respond to auditory features of moderate intensity contained in the instrumental music stimulus, providing a potential mechanism for reliability enhancement. These results parallel findings in V1, where increased contrast sensitivity for visual stimuli and enhanced reliability were also observed following the electrical BF stimulation (Bhattacharyya et al. 2013; De Luna et al. 2017), as well as following the exogenous nicotine application to V1 (Disney et al. 2007). Increases in the sensitivity to tactile stimuli have also been observed in primary somatosensory cortex following the exogenous application of ACh or by mimicking endogenous ACh release through application of acetylcholinesterase inhibitors (Donoghue and Carroll 1987; Oldford and Castro-Alamancos 2003). Directly comparing visual and auditory cortex, it appears that effects of $\mathrm{BF}$ stimulation were more homogenous in visual cortex where only a small fraction of cells exhibit decreases in reliability. Along similar lines, the vast majority of V1 neurons increased their activity following the BF stimulation ( $97 \%$ or $84 / 87$ ), while in A1, there were roughly similar numbers showing increases and decreases $(47 \%$ or $65 / 137,52 \%$ or $72 / 137$, respectively).
Since both sensory systems were studied under isoflurane anesthesia, we can essentially rule out anesthesia as the source of the divergence between visual and auditory reliability effects. We believe that this divergence may reflect modality-specific differences in BF modulation of sensory circuits, consistent with the opposite effects of locomotion on sensory responses that enhance neural responses in the visual cortex while decreasing responses in the auditory cortex (Niell and Stryker 2010; Zhou et al. 2014). Results from A1 also diverged from those in V1 with respect to $B F$ induced gamma activity. While pNB stimulation was effective at desynchronizing A1, as evidenced by a decrease in power at delta frequencies, this was not accompanied by an increase in power at gamma frequencies. This result is consistent with previous studies showing that dorsal A1, the location of most of our recordings, exhibits only very weak gamma activity. Indeed, A1 gamma activity, endogenous or evoked by stimulation of the posterior intralaminar nucleus, appears to be confined to the ventral-posterior border of A1 where it abuts secondary auditory cortex (Sukov and Barth 1998). Taken together, our findings suggest that BF effects encompass modality-specific elements, and that an apparent common regulatory function across sensory modalities is an overall enhancement of cortical reliability, specifically linked to increases in responsivity and enhanced sensitivity.

The BF sends projections to the TRN, which in turn modulates thalamocortical information flow through an inhibitory influence on the MGN. While we sampled only a few cells in the TRN, we did observe both increased and decreased firing in individual neurons in response to auditory stimuli following the electrical pNB stimulation. This is consistent with anatomical and functional evidence indicating both cholinergic and GABAergic projections from BF to TRN (Steriade et al. 1987; Hallanger and Wainer 1988; Asanuma 1989; Jourdain et al. 1989; Asanuma and Porter 1990; Bickford et al. 1994; Thankachan et al. 2019), and we are likely activating both with electrical stimulation. However, it is also possible that, even in the absence of the activation of GABAergic inputs, the dual nature of cholinergic effects, excitatory at the nicotinic receptors and inhibitory at the muscarinic receptors (Ben-Ari et al. 1976; McCormick and Prince 1986; Sun et al. 2013; Pita-Almenar et al. 2014; Sokhadze et al. 2019), could give rise to these mixed results. Nevertheless, since all TRN projections to MGN are inhibitory, we hypothesize that the effect of pNB stimulation on MGN firing rate should also be heterogeneous. This is exactly what we observed in individual MGN neurons, with $42 \%$ (63/149) showing increased and 57\% (86/149) showing decreased responsiveness to auditory stimulation. This finding is consistent with a recent report of no overall firing rate modulation in MGN following the electrical BF stimulation (Sakata 2016), but this may be due to the presence of two functional neuronal subgroups that increase and decrease their firing rates.

Unlike A1, where pNB stimulation enhanced reliability, there was no overall change in the MGN with a similar number of neurons showing significantly increased and decreased reliability. MGN neurons that did increase their reliability showed increases in maximal firing rate as well as an increased sensitivity, i.e., increased firing to less salient stimulus features. MGN neurons that decreased their reliability also showed an increase in maximal firing rate but showed a decrease in sensitivity. We thus hypothesize that enhancement of A1 reliability is in part due to the disinhibition of a population of MGN neurons, brought about by pNB stimulation. This rel+ population of MGN neurons would boost cortical responses to less 
salient stimulus features, whereas the rel-MGN neurons would actually be muted during those stimulus epochs and would therefore contribute little to the cortical response. Indeed, this may explain the cortical bias toward increased reliability and the absence of sensitivity change in cortical rel- units, as this characteristic cannot be inherited from thalamus. Our finding is partially compatible with a computational modeling study, albeit in the visual system, which has focused on this pathway and shown that it can account for enhanced reliability in cortex and thalamus following the BF activation (Avery et al. 2012). The model produces homogenous increases in reliability and general excitation in sensory thalamus, which captures only one aspect of our observations. We suggest that this discrepancy may be due to the absence of cholinergic inputs from BF to TRN in the model. An extension of the model that includes these inputs may offer a more comprehensive view of how the BF can modulate sensory thalamus during different brain states. It is important to remember in this context that BF output pathways may not operate entirely independently but may be coactivated during particular brain states due to common input or local connectivity within the BF (Zant et al. 2016).

A large number of frequency tuned MGN and A1 neurons were affected by pNB stimulation, suggesting that this pathway has a profound impact on auditory information transmission. In both areas, the most common effect was a general, frequencyindependent increase or decrease in firing rate, consistent with a gain modulation of auditory signals. A minority of neurons in both areas exhibited frequency-specific modulation, such that gain changes were restricted to particular frequency bands. This may suggest that the $\mathrm{BF}$ is capable of assigning priority to particular frequencies within the auditory range, possibly according to behavioral demands, as has been suggested to occur between stimuli of different modalities (Wimmer et al. 2015). The BF thalamic projections via the TRN are only one route by which the BF can influence auditory processing, the other being the direct corticopetal projections (Chavez and Zaborszky 2017). The overall similar effects on frequency tuning in MGN and A1 do not allow us to disambiguate between these two modulatory mechanisms, since corticothalamic feedback has also been shown to produce changes in frequency tuning of MGN neurons (Alitto and Usrey 2003). However, a number of our findings argue for an important role in the direct thalamic projections: 1) Up- and downregulation of TRN and MGN activity is difficult to reconcile with a uniquely corticothalamic glutamatergic influence, but consistent with the dual neurotransmitter pathways from pNB to TRN. 2) A significantly larger proportion of MGN cells were modulated by our pNB stimulation than A1 cells, indicating a stronger impact of pNB stimulation on MGN than on A1.3) Granger causality analysis of spiking activity suggests enhancement of feedforward information flow from MGN to A1 following the pNB stimulation. 4) Overall reliability values of spiking activity were higher in MGN than A1, making it unlikely that effects in MGN are simply inherited from A1. These findings suggest an important role in the $\mathrm{BF} \rightarrow$ TRN $\rightarrow$ MGN pathway in modulation of sensory processing.

While our study was largely directed at exploring effects of electrical $\mathrm{BF}$ activation, we also present evidence linking endogenous pNB activation to A1 reliability in awake animals. Gamma oscillation levels in the pNB were negatively correlated with A1 reliability for animals passively listening to instrumental music segments in their home cage. This is consistent with studies linking BF gamma oscillations to default mode regulation (Nair et al. 2018), with periods of high BF gamma being associated with quiet wakefulness and self-directed behaviors such as grooming, whereas during exploratory behaviors, where attention is allocated to external stimuli, BF gamma is greatly reduced. While $\mathrm{BF}$ gamma oscillations are highly conspicuous, their cellular basis is not fully understood. There is evidence that multiple cell types including cholinergic as well as parvalbumin and somatostatin expressing GABAergic neurons play an important role in generation and maintenance of this rhythmic activity (Bartos et al. 2007; Kim et al. 2015; Howe et al. 2017). Further work, for example, involving optogenetic modulation of specific projection pathways, is needed to link cell types and pathways to various functions subserved by the BF.

\section{Funding}

Swiss National Science Foundation (182504 to G.R. and 168602 to A.-L.K.); University of Fribourg.

\section{Conflict of Interest}

None declared.

\section{References}

Alitto HJ, Usrey WM. 2003. Corticothalamic feedback and sensory processing. Curr Opin Neurobiol. 13(4):440-445.

Anaclet C, Pedersen NP, Ferrari LL, Venner A, Bass CE, Arrigoni E, Fuller PM. 2015. Basal forebrain control of wakefulness and cortical rhythms. Nat Commun. 6:8744.

Asanuma C. 1989. Axonal arborizations of a magnocellular basal nucleus input and their relation to the neurons in the thalamic reticular nucleus of rats. Proc Natl Acad Sci U S A. 86(12):4746-4750.

Asanuma C, Porter LL. 1990. Light and electron microscopic evidence for a gabaergic projection from the caudal basal forebrain to the thalamic reticular nucleus in rats. J Comp Neurol. 302(1):159-172.

Avery M, Krichmar JL, Dutt N. 2012. Spiking neuron model of basal forebrain enhancement of visual attention. In: The 2012 International Joint Conference on Neural Networks (IJCNN), Brisbane, QLD, pp. 1-8.

Baghdoyan HA, Spotts JL, Snyder SG. 1993. Simultaneous pontine and basal forebrain microinjections of carbachol suppress rem sleep. J Neurosci. 13(1):229-242.

Bajo VM, Villa AE, de Ribaupierre F, Rouiller EM. 1998. Discharge properties of single neurons in the dorsal nucleus of the lateral lemniscus of the rat. Brain Res Bull. 47(6):595-610.

Barnett L, Seth AK. 2014. The mvgc multivariate granger causality toolbox: a new approach to granger-causal inference. J Neurosci Methods. 223:50-68.

Bartos M, Vida I, Jonas P. 2007. Synaptic mechanisms of synchronized gamma oscillations in inhibitory interneuron networks. Nat Reu Neurosci. 8(1):45-56.

Baxter MG, Chiba AA. 1999. Cognitive functions of the basal forebrain. Curr Opin Neurobiol. 9(2):178-183.

Ben-Ari Y, Dingledine R, Kanazawa I, Kelly JS. 1976. Inhibitory effects of acetylcholine on neurones in the feline nucleus reticularis thalami. J Physiol. 261(3):647-671.

Bhattacharyya A, Veit J, Kretz R, Bondar I, Rainer G. 2013. Basal forebrain activation controls contrast sensitivity in primary visual cortex. BMC Neurosci. 14:55.

Bickford ME, Gunluk AE, Van Horn SC, Sherman SM. 1994. Gabaergic projection from the basal forebrain to the visual 
sector of the thalamic reticular nucleus in the cat. J Comp Neurol. 348(4):481-510.

Boucetta S, Jones BE. 2009. Activity profiles of cholinergic and intermingled gabaergic and putative glutamatergic neurons in the pontomesencephalic tegmentum of urethaneanesthetized rats. J Neurosci. 29(14):4664-4674.

Castro-Alamancos MA. 2002a. Different temporal processing of sensory inputs in the rat thalamus during quiescent and information processing states in vivo. J Physiol. 539(Pt 2):567-578

Castro-Alamancos MA. 2002b. Properties of primary sensory (lemniscal) synapses in the ventrobasal thalamus and the relay of high-frequency sensory inputs. J Neurophysiol. 87(2):946-953.

Chavez C, Zaborszky L. 2017. Basal forebrain cholinergicauditory cortical network: primary versus nonprimary auditory cortical areas. Cereb Cortex. 27(3):2335-2347.

Christophe E, Roebuck A, Staiger JF, Lavery DJ, Charpak S, Audinat E. 2002. Two types of nicotinic receptors mediate an excitation of neocortical layer i interneurons. J Neurophysiol. 88(3):1318-1327.

Clarke PB, Schwartz RD, Paul SM, Pert CB, Pert A. 1985. Nicotinic binding in rat brain: autoradiographic comparison of [3h]acetylcholine, [3h]nicotine, and [125i]-alphabungarotoxin. J Neurosci. 5(5):1307-1315.

Conley M, Kupersmith AC, Diamond IT. 1991. The organization of projections from subdivisions of the auditory cortex and thalamus to the auditory sector of the thalamic reticular nucleus in galago. Eur J Neurosci. 3(11):1089-1103.

Contreras D, Curro Dossi R, Steriade M. 1992. Bursting and tonic discharges in two classes of reticular thalamic neurons. J Neurophysiol. 68(3):973-977.

De Luna P, Veit J, Rainer G. 2017. Basal forebrain activation enhances between-trial reliability of low-frequency local field potentials (LFP) and spiking activity in tree shrew primary visual cortex (v1). Brain Struct Funct. 222(9):4239-4252.

Deschenes M, Roy JP, Steriade M. 1982. Thalamic bursting mechanism: an inward slow current revealed by membrane hyperpolarization. Brain Res. 239(1):289-293.

Detari L, Rasmusson DD, Semba K. 1997. Phasic relationship between the activity of basal forebrain neurons and cortical eeg in urethane-anesthetized rat. Brain Res. 759(1):112-121.

Disney AA, Aoki C, Hawken MJ. 2007. Gain modulation by nicotine in macaque v1. Neuron. 56(4):701-713.

Donoghue JP, Carroll KL. 1987. Cholinergic modulation of sensory responses in rat primary somatic sensory cortex. Brain Res. 408(1-2):367-371.

Edeline JM, Hars B, Maho C, Hennevin E. 1994. Transient and prolonged facilitation of tone-evoked responses induced by basal forebrain stimulations in the rat auditory cortex. Exp Brain Res. 97(3):373-386.

Eggermann E, Feldmeyer D. 2009. Cholinergic filtering in the recurrent excitatory microcircuit of cortical layer 4. Proc Natl Acad Sci U S A. 106(28):11753-11758.

Goard M, Dan Y. 2009. Basal forebrain activation enhances cortical coding of natural scenes. Nat Neurosci. 12(11):1444-1449.

Gulledge AT, Park SB, Kawaguchi Y, Stuart GJ. 2007. Heterogeneity of phasic cholinergic signaling in neocortical neurons. J Neurophysiol. 97(3):2215-2229.

Halassa MM, Chen Z, Wimmer RD, Brunetti PM, Zhao S, Zikopoulos B, Wang F, Brown EN, Wilson MA. 2014. Statedependent architecture of thalamic reticular subnetworks. Cell. 158(4):808-821.
Hallanger AE, Wainer BH. 1988. Ultrastructure of chatimmunoreactive synaptic terminals in the thalamic reticular nucleus of the rat. J Comp Neurol. 278(4):486-497.

Harris KD, Thiele A. 2011. Cortical state and attention. Nat Rev Neurosci. 12(9):509-523.

Hasselmo ME, McGaughy J. 2004. High acetylcholine levels set circuit dynamics for attention and encoding and low acetylcholine levels set dynamics for consolidation. Prog Brain Res. 145:207-231.

Herrero JL, Roberts MJ, Delicato LS, Gieselmann MA, Dayan P, Thiele A. 2008. Acetylcholine contributes through muscarinic receptors to attentional modulation in v1. Nature. 454(7208):1110-1114.

Hirata A, Aguilar J, Castro-Alamancos MA. 2006. Noradrenergic activation amplifies bottom-up and top-down signal-tonoise ratios in sensory thalamus. J Neurosci. 26(16):4426-4436.

Hirata A, Castro-Alamancos MA. 2011. Effects of cortical activation on sensory responses in barrel cortex. J Neurophysiol. 105(4):1495-1505.

Howe WM, Gritton HJ, Lusk NA, Roberts EA, Hetrick VL, Berke JD, Sarter M. 2017. Acetylcholine release in prefrontal cortex promotes gamma oscillations and theta-gamma coupling during cue detection. J Neurosci. 37(12):3215-3230.

Jahnsen H, Llinas R. 1984. Electrophysiological properties of Guinea-pig thalamic neurones: an in vitro study. J Physiol. 349:205-226.

Jimenez-Capdeville ME, Dykes RW, Myasnikov AA. 1997. Differential control of cortical activity by the basal forebrain in rats: a role for both cholinergic and inhibitory influences. J Comp Neurol. 381(1):53-67.

Jones EG. 1975. Some aspects of the organization of the thalamic reticular complex. J Comp Neurol. 162(3):285-308.

Jourdain A, Semba K, Fibiger HC. 1989. Basal forebrain and mesopontine tegmental projections to the reticular thalamic nucleus: an axonal collateralization and immunohistochemical study in the rat. Brain Res. 505(1):55-65.

Kang JI, Groleau M, Dotigny F, Giguere H, Vaucher E. 2014. Visual training paired with electrical stimulation of the basal forebrain improves orientation-selective visual acuity in the rat. Brain Struct Funct. 219(4):1493-1507.

Kang JI, Vaucher E. 2009. Cholinergic pairing with visual activation results in long-term enhancement of visual evoked potentials. PLoS One. 4(6):e5995.

Kawai H, Lazar R, Metherate R. 2007. Nicotinic control of axon excitability regulates thalamocortical transmission. Nat Neurosci. 10(9):1168-1175.

Kim JH, Jung AH, Jeong D, Choi I, Kim K, Shin S, Kim SJ, Lee SH. 2016. Selectivity of neuromodulatory projections from the basal forebrain and locus ceruleus to primary sensory cortices. J Neurosci. 36(19):5314-5327.

Kim T, Thankachan S, McKenna JT, McNally JM, Yang C, Choi JH, Chen L, Kocsis B, Deisseroth K, Strecker RE et al. 2015. Cortically projecting basal forebrain parvalbumin neurons regulate cortical gamma band oscillations. Proc Natl Acad Sci U S A. 112(11):3535-3540.

Kruglikov I, Rudy B. 2008. Perisomatic gaba release and thalamocortical integration onto neocortical excitatory cells are regulated by neuromodulators. Neuron. 58(6):911-924.

Laplante F, Morin Y, Quirion R, Vaucher E. 2005. Acetylcholine release is elicited in the visual cortex, but not in the prefrontal cortex, by patterned visual stimulation: a dual in vivo microdialysis study with functional correlates in the rat brain. Neuroscience. 132(2):501-510. 
Lavine N, Reuben M, Clarke PB. 1997. A population of nicotinic receptors is associated with thalamocortical afferents in the adult rat: Laminal and areal analysis. J Comp Neurol. 380(2):175-190.

Levey AI, Hallanger AE, Wainer BH. 1987. Cholinergic nucleus basalis neurons may influence the cortex via the thalamus. Neurosci Lett. 74(1):7-13.

Llinas RR, Steriade M. 2006. Bursting of thalamic neurons and states of vigilance. J Neurophysiol. 95(6):3297-3308.

Losier BJ, Semba K. 1993. Dual projections of single cholinergic and aminergic brainstem neurons to the thalamus and basal forebrain in the rat. Brain Res. 604(1-2):41-52.

Marguet SL, Harris KD. 2011. State-dependent representation of amplitude-modulated noise stimuli in rat auditory cortex. $J$ Neurosci. 31(17):6414-6420.

McCormick DA. 1993. Actions of acetylcholine in the cerebral cortex and thalamus and implications for function. Prog Brain Res. 98:303-308.

McCormick DA, Prince DA. 1986. Acetylcholine induces burst firing in thalamic reticular neurones by activating a potassium conductance. Nature. 319(6052):402-405.

McCormick DA, Prince DA. 1987. Actions of acetylcholine in the Guinea-pig and cat medial and lateral geniculate nuclei, in vitro. J Physiol. 392:147-165.

Mesulam MM, Mufson EJ, Wainer BH, Levey AI. 1983. Central cholinergic pathways in the rat: an overview based on an alternative nomenclature (ch1-ch6). Neuroscience. 10(4):1185-1201.

Munoz W, Rudy B. 2014. Spatiotemporal specificity in cholinergic control of neocortical function. Curr Opin Neurobiol. 26:149-160.

Nair J, Klaassen AL, Arato J, Vyssotski AL, Harvey M, Rainer G. 2018. Basal forebrain contributes to default mode network regulation. Proc Natl Acad Sci U S A. 115(6):1352-1357.

Nair J, Klaassen AL, Poirot J, Vyssotski A, Rasch B, Rainer G. 2016. Gamma band directional interactions between basal forebrain and visual cortex during wake and sleep states. J Physiol. 110(1-2):19-28.

Niell CM, Stryker MP. 2010. Modulation of visual responses by behavioral state in mouse visual cortex. Neuron. 65(4):472-479.

Oldford E, Castro-Alamancos MA. 2003. Input-specific effects of acetylcholine on sensory and intracortical evoked responses in the "barrel cortex" in vivo. Neuroscience. 117(3):769-778.

Parent A, Pare D, Smith Y, Steriade M. 1988. Basal forebrain cholinergic and noncholinergic projections to the thalamus and brainstem in cats and monkeys. J Comp Neurol. 277(2):281-301.

Pinto L, Goard MJ, Estandian D, Xu M, Kwan AC, Lee SH, Harrison TC, Feng GP, Dan Y. 2013. Fast modulation of visual perception by basal forebrain cholinergic neurons. Nat Neurosci. 16(12):1857-1863.

Pita-Almenar JD, Yu D, Lu HC, Beierlein M. 2014. Mechanisms underlying desynchronization of cholinergic-evoked thalamic network activity. J Neurosci. 34(43):14463-14474.

Quinn LK, Nitz DA, Chiba AA. 2010. Learning-dependent dynamics of beta-frequency oscillations in the basal forebrain of rats. Eur J Neurosci. 32(9):1507-1515.

Rotter A, Birdsall NJ, Burgen AS, Field PM, Hulme EC, Raisman G. 1979. Muscarinic receptors in the central nervous system of the rat. I. Technique for autoradiographic localization of the binding of [3h]propylbenzilylcholine mustard and its distribution in the forebrain. Brain Res. 180(2):141-165.
Rye DB, Wainer BH, Mesulam MM, Mufson EJ, Saper CB. 1984. Cortical projections arising from the basal forebrain: a study of cholinergic and noncholinergic components employing combined retrograde tracing and immunohistochemical localization of choline acetyltransferase. Neuroscience. 13(3):627-643.

Sakata S. 2016. State-dependent and cell type-specific temporal processing in auditory thalamocortical circuit. Sci Rep. 6:18873.

Schmitt LI, Wimmer RD, Nakajima M, Happ M, Mofakham S, Halassa MM. 2017. Thalamic amplification of cortical connectivity sustains attentional control. Nature. 545(7653):219-223.

Semba K, Reiner PB, McGeer EG, Fibiger HC. 1988. Brainstem afferents to the magnocellular basal forebrain studied by axonal transport, immunohistochemistry, and electrophysiology in the rat. J Comp Neurol. 267(3):433-453.

Sherman SM. 2001a. Tonic and burst firing: dual modes of thalamocortical relay. Trends Neurosci. 24(2):122-126.

Sherman SM. 2001b. A wake-up call from the thalamus. Nat Neurosci. 4(4):344-346.

Shosaku A, Sumitomo I. 1983. Auditory neurons in the rat thalamic reticular nucleus. Exp Brain Res. 49(3):432-442.

Smith WJ, Stewart J, Pfaus JG. 1997. Tail pinch induces fos immunoreactivity within several regions of the male rat brain: effects of age. Physiol Behav. 61(5):717-723.

Sokhadze G, Campbell PW, Guido W. 2019. Postnatal development of cholinergic input to the thalamic reticular nucleus of the mouse. Eur J Neurosci. 49(8):978-989.

Sottile SY, Hackett TA, Cai R, Ling L, Llano DA, Caspary DM. 2017. Presynaptic neuronal nicotinic receptors differentially shape select inputs to auditory thalamus and are negatively impacted by aging. J Neurosci. 37(47):11377-11389.

Steriade M, Datta S, Pare D, Oakson G, Curro Dossi RC. 1990. Neuronal activities in brain-stem cholinergic nuclei related to tonic activation processes in thalamocortical systems. J Neurosci. 10(8):2541-2559.

Steriade M, Parent A, Pare D, Smith Y. 1987. Cholinergic and noncholinergic neurons of cat basal forebrain project to reticular and mediodorsal thalamic nuclei. Brain Res. 408(1-2): 372-376.

Sukov W, Barth DS. 1998. Three-dimensional analysis of spontaneous and thalamically evoked gamma oscillations in auditory cortex. J Neurophysiol. 79(6):2875-2884.

Sun YG, Pita-Almenar JD, Wu CS, Renger JJ, Uebele VN, Lu HC, Beierlein M. 2013. Biphasic cholinergic synaptic transmission controls action potential activity in thalamic reticular nucleus neurons. J Neurosci. 33(5):2048-2059.

Swanson LW, Mogenson GJ, Gerfen CR, Robinson P. 1984. Evidence for a projection from the lateral preoptic area and substantia innominata to the 'mesencephalic locomotor region' in the rat. Brain Res. 295(1):161-178.

Swanson LW, Mogenson GJ, Simerly RB, Wu M. 1987. Anatomical and electrophysiological evidence for a projection from the medial preoptic area to the 'mesencephalic and subthalamic locomotor regions' in the rat. Brain Res. 405(1): 108-122.

Thankachan S, Katsuki F, McKenna JT, Yang C, Shukla C, Deisseroth K, Uygun DS, Strecker RE, Brown RE, McNally JM et al. 2019. Thalamic reticular nucleus parvalbumin neurons regulate sleep spindles and electrophysiological aspects of schizophrenia in mice. Sci Rep. 9(1):3607.

Vanderwolf CH. 1988. Cerebral activity and behavior: control by central cholinergic and serotonergic systems. Int Rev Neurobiol. 30:225-340. 
Wimmer RD, Schmitt LI, Davidson TJ, Nakajima M, Deisseroth K, Halassa MM. 2015. Thalamic control of sensory selection in divided attention. Nature. 526(7575):705-709.

Xu M, Chung S, Zhang S, Zhong P, Ma C, Chang WC, Weissbourd B, Sakai N, Luo L, Nishino S et al. 2015. Basal forebrain circuit for sleep-wake control. Nat Neurosci. 18(11):1641-1647.

Yu AJ, Dayan P. 2002. Acetylcholine in cortical inference. Neural Netw. 15(4-6):719-730.

Zaborszky L, Csordas A, Mosca K, Kim J, Gielow MR, Vadasz C, Nadasdy Z. 2015. Neurons in the basal forebrain project to the cortex in a complex topographic organization that reflects corticocortical connectivity patterns: an experimental study based on retrograde tracing and $3 \mathrm{~d}$ reconstruction. Cereb Cortex. 25(1):118-137.
Zant JC, Kim T, Prokai L, Szarka S, McNally J, McKenna JT, Shukla C, Yang C, Kalinchuk AV, McCarley RW et al. 2016. Cholinergic neurons in the basal forebrain promote wakefulness by actions on neighboring non-cholinergic neurons: an opto-dialysis study. J Neurosci. 36(6):2057-2067.

Zhou M, Liang F, Xiong XR, Li L, Li H, Xiao Z, Tao HW, Zhang LI. 2014. Scaling down of balanced excitation and inhibition by active behavioral states in auditory cortex. Nat Neurosci. 17(6):841-850.

Zinke W, Roberts MJ, Guo K, McDonald JS, Robertson R, Thiele A. 2006. Cholinergic modulation of response properties and orientation tuning of neurons in primary visual cortex of anaesthetized marmoset monkeys. Eur J Neurosci. 24(1): 314-328. 